\title{
Three-dimensional Simulation of Thermal Harmonic Lasing FEL with Detuning of the Fundamental
}

\author{
E. Salehi ${ }^{(1)}$, B. Maraghechi ${ }^{(1,2)}$ 网 and, N. S. Mirian ${ }^{(2,3)}$ \\ (1)Department of Physics, Amirkabir University of Technology, \\ Postal code 15875-4413, Tehran, Iran. \\ (2) School of Particle and Accelerator Physics, \\ Institute for Research in Fundamental Sciences (IPM), \\ Postal code: 19395-5531, Tehran, Iran and \\ (3) UVSOR Facility (UVSOR) Institute for Molecular Science, \\ 444-8585, Myodaiji J - Okazaki, japan.
}

\begin{abstract}
Detuning of the fundamental is a way to enhance harmonic generation . By this method, the wiggler is composed of two segments in such a way that the fundamental resonance of the second segment to coincide with the third harmonic of the first segment of the wiggler to generate extreme ultraviolet radiation and x-ray emission. A set of coupled, nonlinear, and first-order differential equations in three dimensions describing the evolution of the electron trajectories and the radiation field with warm beam is solved numerically by CYRUS 3D code in the steady-state for two models (1) seeded free electron laser (FEL) and (2) shot noise on the electron beam (self-amplified spontaneous emission FEL). Thermal effects in the form of longitudinal velocity spread is considered. Three-dimensional simulation describes self-consistently the longitudinal spatial dependence of radiation waists, curvatures, and amplitudes together with the evaluation of the electron beam. The evolutions of the transverse modes are investigated for the fundamental resonance and the third harmonic. Also, the effective modes of the third harmonic are studied. In this paper, we found that detuning of the fundamental with shot noise gives more optimistic result than the seeded FEL.
\end{abstract}

${ }^{*}$ Electronic address: behrouz@aut.ac.ir 


\section{INTRODUCTION}

Fourth generation light sources have given us the capability of exploring the molecular and atomic phenomena. High-gain free electron laser (FEL) amplifiers hold great prospects of reaching coherent high power radiation in the x-ray region of the electromagnetic spectrum. In recent years, a great effort of researchers has been devoted to study the process of higher harmonic generation in achieving lasing at shorter wavelengths. A possible way for obtaining $\mathrm{x}$-ray wavelength is by using nonlinear harmonic generation [1-6].

Radiation of the electron beam in the planar wiggler contains odd harmonics but the output power at the $h$ th harmonic is rather small and is of the order of $10^{-h}$ times the power of the fundamental [1, 3, 5, 7]. Various schemes have been proposed to enhance the harmonic intensity, these are high-gain harmonic generation [8 12], echo-enabled harmonic generation using the beam echo effect [13, 14], and the two-beam FEL for frequency upconversion [15 17]. Another technique is two harmonic undulators in which an on axis field oscillates with integer multiples of spatial periods and with different orthogonal polarizations [18]. The analysis of this method is achieved with PROMETEO code developed by Dattoli and coworkers [19], which has been benchmarked with MEDUSA. Recently, McNeil et al. [20] proposed a harmonic lasing method for FEL amplifiers that can amplify the higher harmonics by suppressing the interaction at the fundamental resonance. They showed that this configuration can significantly extend the operation band of user facilities.

Reference [20] has outlined two methods for suppressing the interaction at the fundamental resonance while allowing the third harmonic to evolve to saturation. The first method is based upon the shifting of the phase of the fundamental between the wiggler segments, which can be controlled by various techniques [21]. For the $h$ th harmonic, this phase shift should be $2 \pi n / h$, where $n=1,2,3, \ldots$ is an integer number and $h=3,5,7, \ldots$ is the harmonic number. Freund and coworkers [22] investigated the effects of varying the gap lengths between the wiggler segments and varying the electron beam $\beta$ function on the third harmonic generation with phase shift of $2 \pi / 3$. The second method is the detuning of the fundamental by considering two different segments for the wiggler. Two segments of the wiggler have different magnetic field intensity while the wiggler period, $\lambda_{w}$, and the initial average electron beam energy, $\gamma$, are kept constant. It has been suggested in Ref. [21] that a combined use of the phase shifting and detuning of the fundamental can reduce the bandwidth and increase 
the brilliance of the x-ray beam at saturation. In Ref. [23], detuning of the fundamental has been compared for the nonaveraged and averaged methods in one-dimension and it is shown that there is a remarkable agreement between the averaged and nonaveraged simulations for the evolution of the third harmonic.

The method of detuning of fundamental has not been studied in three-dimensions in the nonaveraged procedure. The 3D averaged wiggler method for detuning of the fundamental method was studied in [21]; they showed that the gain length of detuning for the fundamental is larger than that of the phase shift method (in the case of negligible energy spread) and this result is consistent with the 1D theory [20]. They also showed that 3D effects actually improve the situation and makes harmonic lasing even more attractive compared to the 1D theory. In this paper, three-dimensional features such as diffraction, radiation guiding, and the evolution of transverse mode have been considered. The guiding of laser light by an electron beam (optical guiding) in an FEL occurs in the exponential gain regime [24 26] when the coherent interaction between the source electron beam and the electromagnetic field introduces an inward curvature in the phase front of the light, which refracts it back toward the lasing core of the electron beam. During the amplification, the electron beam that propagates through the wiggler operates simultaneously as a guiding structure that suppresses the diffraction, reducing the transverse power losses, and enhancing the electromagnetic field amplification. The evolution of transverse modes is important in planning for the future user facilities that intend to employ radiation from this system [27].

The thermal effect of the electron beam is particularly important for higher harmonics, because they are more sensitive to the energy spread compared to the fundamental [7, 28, 30]. The energy spread is considered as a Gaussian energy distribution in MEDUSA code for nonlinear harmonic generation [29]. Also, in reference [21], the energy spread is considered and the gain length for the detuning of the fundamental is compared with the third harmonic in conventional FEL. The effect of optimizing the $\beta$ function at the third harmonic in the phase shift method is investigated in Ref. [22] by considering the thermal effects, where the $\beta$ function is adjusted depending on to the energy spread. In this paper, we consider the thermal effects only in the longitudinal direction and ignore thermal effects in the transverse momentum, because the longitudinal spread is more effective than a transverse spread in reducing the growth rate [31, 32].

The initial radiation signal in a single-pass amplifier configuration based upon the prin- 
ciple of self-amplified spontaneous emission (SASE) originates from the temporally random microbunching present on the electron beam as they enter the wiggler. SASE amplifiers start up from the spontaneous emission due to shot noise on the electron beam, which is due to the random fluctuations in the phase distribution of the electrons [33 35]. The seed power for the fundamental overwhelms the shot noise, whereas it treats correctly in order to model the initial start up of harmonic radiation. Penman and McNeil discussed a particular algorithm used in one-dimensional (1D) approximation (in their 1D code) in order to simulate FEL start up noise [36]. In Ref. [37, the shot noise algorithm is obtained for multidimensional FEL simulation codes. Freund and coworkers [39] discussed and compared two different shot noise models that are implemented in both 1D wiggler-averaged (PERSEO) and non-wiggler-averaged (MEDUSA 1D) simulation codes, and a 3D non-wiggler-averaged (MEDUSA) formulation. They found out that there is a very good agreement between PERSEO and MEDUSA 1D for the evolution at the fundamental and harmonics. They also showed that the harmonics saturate slightly prior to the saturation point of the fundamental. Also, the high gain FEL, in the SASE regime, operating with segmented undulators is studied in Ref. 38] using a set of semianalytical formulas, with the effect of the energy spread taken into account.

The aim of this paper is to present a three-dimensional simulation of the emission at the fundamental and third harmonic in the non-wiggler-averaged-orbit approximation of the harmonic lasing FEL with source-dependent expansion[40 43]. Therefore, the source function is incorporated self-consistently into the functional dependence of the radiation waist, the radiation wavefront curvature, and the radiation amplitude instead of using the usual modal expansion consisting of vacuum Laguerre-Gaussian or Hermite-Gaussian functions. It is important to emphasize that no wiggler average is imposed on the orbit equations. Therefore, It is possible to treat the injection of the beam into the wiggler, with the ease of inclusion of external focusing or dispersive magnetic components in the beam line and the facility for using an actual magnetic field in the numerical solution. The third harmonic lasing is considered so that the operating wavelength is in the EUV domain. The slippage of the radiation with respect to the long electron bunch is ignored. Also, we present numerical results for the fundamental resonance and the third harmonic including shot noise on the electron beam. We found that in many cases, SASE FEL gives more optimistic result than seeded FEL in detuning of the fundamental method. For example, one of the results of 
our studies is that the lowest mode in shot noise is more effective at the saturation point compared to that in the seeded FEL.

The code for this purpose is named CYRUS 3D, which was developed by PhD students in Amirkabir University and Institute for Research in Fundamental Sciences (IPM)[44]. This code follows MEDUSA 3D [29] formulation.

The organization of this paper is as follows. The simulations conducted using the CYRUS 3D simulation code is briefly described in Sec. II. A general discussion of the retuned wiggler parameter is given in Sec. III. The simulation results for the fundamental resonance and the third harmonic in this system for seeded FEL and SASE FEL are discussed in Sec. IV. In Sec. V, the summary and discussion are presented.

\section{DESCRIPTION OF THE SIMULATION CODE}

The simulation code for three-dimensional non-wiggler averaged-orbit formulation is CYRUS 3D code, which is written in standard Fortran 95. This code is time independent with harmonics and thermal effects taken into account. It models planar wiggler generated by magnets with parabolically shaped pole faces for additional focusing; hence, we can write

$$
\begin{aligned}
\mathbf{B}_{\mathrm{w}}(\mathbf{X})=B_{w}(z)\left\{\cos \left(k_{w} z\right)\left[\hat{\mathbf{e}}_{x} \sinh \left(\frac{k_{w} x}{\sqrt{2}}\right) \sinh \left(\frac{k_{w} y}{\sqrt{2}}\right)+\hat{\mathbf{e}}_{y} \cosh \left(\frac{k_{w} x}{\sqrt{2}}\right) \cosh \left(\frac{k_{w} y}{\sqrt{2}}\right)\right]\right. \\
\left.\quad-\sqrt{2} \hat{\mathbf{e}}_{z} \sin \left(k_{w} z\right) \cosh \left(\frac{k_{w} x}{\sqrt{2}}\right) \sinh \left(\frac{k_{w} y}{\sqrt{2}}\right)\right\},
\end{aligned}
$$

where $B_{w}$ represents the wiggler amplitude, $k_{w}=2 \pi / \lambda_{w}$ is the wiggler wave number corresponding to the wiggler period $\lambda_{w}$. This type of wiggler has enough focusing on an electron beam in both directions in the plane transverse. And the electromagnetic field is represented as a superposition of Gauss-Hermit modes in the slowly varying amplitude approximation. The vector potential of the linearly polarized radiation field is

$$
\mathbf{A}_{r}(\mathbf{X}, t)=\sum_{l, n, h} \frac{1}{2} e_{l, n, h}(x, y) e^{i \alpha_{h} \mathrm{r}^{2} / \mathrm{w}_{\mathrm{h}}^{2}} \delta A_{l, n, h} e^{i h(k z-\omega t)} \hat{\mathbf{e}_{x}}+c . c .,
$$

where the summation indices $l$ and $n$ denote the transverse mode structure, $h$ is the harmonic

number and $e_{l, n, h}(x, y)=e^{-r^{2} / w_{h}^{2}} H_{l}\left(\sqrt{2} x / w_{h}\right) H_{n}\left(\sqrt{2} y / w_{h}\right)$ is the transverse structure of each 
mode. Here, $H_{l}$ is the Hermite polynomial of order $l, \omega_{h}$ is the spot size of the $h$ th harmonic, $\alpha_{h}$ is related to the curvature of the phase front, $\omega$ is the fundamental frequency, and c.c. denotes the complex conjugate. Electron trajectories are integrated using the threedimensional (3D) Lorentz force equations in the magnetostatic and electromagnetic fields. It is important to emphasize that no average is performed over the Lorentz force equation. So, Cyrus can simulate the injection of the electrons into each segment of the wiggler.

This code like MEDUSA 3D employs nonaverage equations. The details of the formulation is explained in Ref. [29]. We simulate the detuning of the fundamental FEL in which the wiggler consists of two segments. In the harmonic lasing FEL the wiggler segments have two different magnetic field strengths but the same wavelength $\lambda_{w}$.

The thermal effect of the electron beam on the harmonic gain is particularly important. The kinetic theory has shown that the thermal FEL lowers the growth rate [31, 45]. Higher harmonics are more sensitive to the energy spread than the fundamental one [4, 20, 28, It was concluded in Ref. [20] that harmonic lasing with phase shifting is more sensitive to the emittance and the energy spread than the harmonic lasing with detuning of the fundamental. In Refs. [32, 46], a spread in the traverse momentum with constant total energy is considered. They showed that a longitudinal spread is more effective than a transverse spread in reducing the growth rate.

To consider effects of the energy spread, we assume longitudinal spread without any spread in the transverse momentum. So, the initial conditions is chosen to model the axial injection of the electron beam with the energy in the form of a Gaussian distribution function that is peaked around the initial energy of the beam. We choose the thermal distribution function as

$$
G_{0}\left(p_{z}\right)=\sqrt{\frac{2}{\pi}} \frac{1}{\Delta p_{z}} \exp \left(-\frac{2\left(p_{z}-p_{z 0}\right)^{2}}{\Delta p_{z^{2}}}\right),
$$

where $p_{0}$ and $\Delta p_{z 0}$ are the initial bulk momentum and momentum spread, respectively. The corresponding axial energy spread can be written as

$$
\frac{\Delta \gamma_{0}}{\gamma_{0}}=\frac{\gamma_{0}^{2}-1}{\gamma_{0}^{2}} \frac{\Delta p_{z 0}}{p_{0}}
$$


where $\gamma=\sqrt{1+p_{0}^{2} / m_{e}^{2} c^{4}}$ is the average initial energy of electrons at the injection time. Therefore, the averaging operator in Eq. 10 of Ref. [29] is defined by

$$
\langle(\cdots)\rangle=\int \frac{d \psi_{0}}{2 \pi} \sigma_{\|}\left(\psi_{0}\right) \iint d x_{0} d y_{0} \sigma_{\perp}\left(x_{0}, y_{0}\right) \int d p G_{0}\left(p_{z}\right)(\cdots) .
$$

Shot noise is a random component in the initial phase $\psi_{0}$ of the macroparticles in such a way that $\left|\left\langle\exp \left(i \psi_{0}\right)\right\rangle\right|=1 / \sqrt{N_{e}}$, where $N_{e}$ is the number of correlated electrons. In the steady-state simulation, each beamlet interacts with the electromagnetic field in an identical manner so that the simulation treats only one beamlet. Therefore, the number of electrons per beamlet $n_{e}$ is given by

$$
n_{e}=\frac{I_{b} \lambda}{e v_{b}}
$$

where $I_{b}$ is the beam current, $\lambda$ is the wavelength, $e$ is the electron charge, and $v_{b}$ is the bulk axial beam velocity. Since the total number of interacting electrons $N_{e}$ will include contribution from multiple slices it will be given approximately by [47]

$$
N_{e}=4.3 \frac{L_{g}}{\lambda_{w}} n_{e}
$$

where $L_{g}$ is the field exponentiation length.

In order to introduce shot noise, a perturbation with the desired harmonic content is added to the initial phase $\left\{\psi_{0 j}\right\}$ to obtain a distribution of $\left\{\psi_{0 j}^{\prime}\right\}$, which can be written as 39

$$
\psi_{0 j}^{\prime}=\psi_{0 j}+\sum_{h} \delta \psi_{h} \sin \left[h\left(\psi_{0 j}-\varphi\right)\right],
$$

where $\delta \psi_{h} \ll 1$ is chosen to describe the Poisson statistics and $\varphi$ is chosen randomly over the interval $[0,2 \pi]$. By Choosing $h \delta \psi_{h}=\delta \psi_{1}$ and $\delta \psi_{1}=2 / \sqrt{N_{e}}$ we will have

$$
\left|\left\langle\exp \left(i h \psi_{0}^{\prime}\right)\right\rangle\right|=\frac{1}{2} \delta \psi_{1}=\frac{1}{\sqrt{N_{e}}},
$$

and the correct Poisson statistics is recovered. 


\section{RETUNED WIGGLER PARAMETER}

We consider the retuned fundamental resonant wavelength by changes in the wiggler magnetic field alone. The wiggler is composed of two segments and the wavelength of the fundamental resonance of the second segment is decreased by reducing the magnetic field strength of the second segment of the wiggler. In this case, for the first segment, the rms wiggler parameter is $a_{1}$ and the fundamental resonant wavelength is $\lambda_{1}$ giving the harmonic resonant wavelengths as $\lambda_{h}=\lambda_{1} / h, h=3,5,7, \ldots$ In the second segment, the rms wiggler parameter is reset to $a_{n}$ so that the new resonant fundamental wavelength is the $n$th harmonic of the first segment, $\lambda_{1}^{\prime}=\lambda_{n}$. For the assumed fixed beam energy and wiggler period, the retuned wiggler parameter $a_{n}$ is obtain from the FEL resonance relation

$$
\frac{1+a_{1}^{2}}{1+a_{n}^{2}}=n
$$

Obviously, $a_{1}$ must be larger than $a_{c}=\sqrt{n-1}$ as there are no real solutions for $a_{n}$ for $a_{1}<a_{c}$. So, the wiggler can not be reduced to a fundamental wavelength $\lambda_{1}^{\prime}=\lambda_{n}$ for $a_{1}<a_{c}$. We consider tuning the harmonic interaction by decreasing the wiggler magnetic field; it is clear that this is impractical for an operating X-ray FEL.

\section{NUMERICAL ANALYSIS}

Self-consistent first-order nonlinear differential equations are solved numerically using the fourth-order Runge-Kutta algorithm subject to the appropriate initial conditions and in the time independent approximation where the pulse length is much longer than the slippage length over the course of the wiggler. The particle averages are carried out using a Gaussian quadrature technique in each of the degrees of freedom $\left(x_{0}, y_{0}, \psi_{0}, \varphi_{0}, p_{z 0}, \gamma_{0}\right)$. The number of Gauss-Hermite modes that are needed in the code depends on each particular example. The self-guiding effects of the electron beam in an FEL during exponential gain become dominant over the diffraction, and the balance depends on the Rayleigh length, the growth rate, and the evolution of the beam envelope. Therefore, it is necessary to choose a suitable basis set in order to determine the optical mode content. The number of modes that are determined by an empirical procedure in which successive simulation runs are made with an increasing number of modes until convergence of the saturation power and saturation length are achieved. 
The parameters for the electron beam, the wiggler, and the radiation in the simulation are as follows. The electron beam has the relativistic factor of 964 , a peak current of 300 $A$, an initial radius of $0.01495 \mathrm{~cm}$, and an energy spread of $0.1 \%$. The wiggler period is 3.3 $\mathrm{cm}$ and exhibits a peak of on-axis amplitude equal to $10.06 \mathrm{KG}$. An entry taper region is $N_{w}=10$ wiggler periods in length which is necessary in order to inject the electrons into the steady-state trajectories. Using these beams and wiggler parameters, the fundamental resonance is at a wavelength of $102.9 \mathrm{~nm}$ in the 1D resonance formula. Because of the betatron motion in three dimensions, fundamental resonance is found at the wavelength of $103.6 \mathrm{~nm}$, which is seeded with a $10 \mathrm{~W}$ of optical power. The third harmonic wavelength is at $34.5 \mathrm{~nm}$ and starts from zero initial power. The initial radiation waists are $0.037 \mathrm{~cm}$ and the initial alpha parameters are chosen to be zero. The initial state of electron beams is chosen to model the injection of an axisymmetric electron beams with the flattop density profiles, i.e., $\sigma_{\perp}=1$. For unbunched electron beam, the particles are uniformly distributed in phase.

In this case, we assume a seed power for the fundamental of $10 \mathrm{KW}$ and include the third harmonic, which starts from zero initial power. The fundamental resonance is suppressed by reducing the wiggler magnetic field strength at $L_{1}=10 \mathrm{~m}$ with $a_{3}=0.97$ while the third harmonic grows to saturation. In Ref. [23], it has been shown that the saturation power and saturation length of the third harmonic radiation depends on the length of the first segment of the wiggler $L_{1}$. Since the main bunching for the electron beam in the first segment of the wiggler corresponds to the fundamental resonance the electron beam in the beginning of the second segment of the wiggler, in which the third harmonic of the first segment of the wiggler is a seed for the second segment, is not uniformly distributed. This difference in the distribution of the electrons at different lengths of the first segment of the wiggler leads to changes in the saturation power and saturation length in the second segment of the wiggler, compared to the conventional wiggler. The optimum length of the first segment of the wiggler is determined by a successive runs of the code until an optimized saturation power and saturation length of the third harmonic are obtained.

In Fig. 1, the power of the fundamental resonance (solid line) and the third harmonic (dashed line) are plotted as functions of the distance through the wiggler. The intensity of the shorter wavelength is larger than the intensity of the fundamental wavelength. This means that by reducing the wiggler magnetic field, the fundamental resonance will be suppressed 


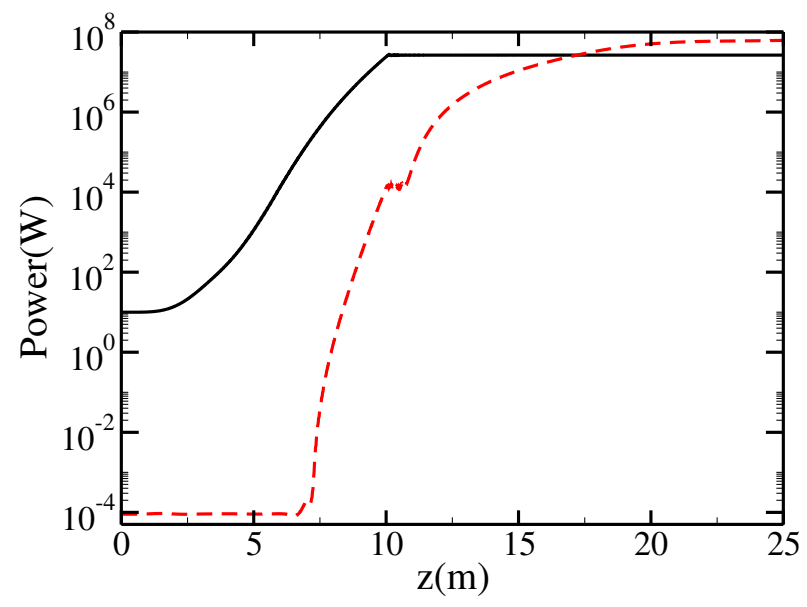

Figure 1: Evolution for the power for the fudamental resonance (solid line) and the third harmonic (dashed line).

and the third harmonic of the first segment of the wiggler is a seed for the fundamental harmonic of the second segment of the wiggler leading to higher power. The fundamental resonance in Fig. 1 is suppressed at $z=10 \mathrm{~m}$ with the power of $2.7 \times 10^{7} \mathrm{~W}$. The third harmonic has three distinct regimes, a small gain regime that ends at $z=6.6 \mathrm{~m}$, exponential growth, and approach to the saturation point at $z=21.5 \mathrm{~m}$ with the power of $4.8 \times 10^{7} \mathrm{~W}$.

Evolution of the radiation amplitude in the transverse plane is shown in Figs. 2(a) and 2 (b) as a function of $\mathrm{z}$ for the fundamental mode and the third harmonic, respectively. Due to the normalization of transverse profile to peak intensity of 1 , these figures do not show the amplification of the radiation; however, they show that the profile of the amplitude of radiation in the transverse plane gets narrower when the radiation moves toward the saturation point. It needs to be mentioned that this mode narrowing is stronger for the third harmonic. Figure 2(b) shows that the transverse intensity profile of the third harmonic initially widens up to the point where the small signal gain ends at $z=6.6 \mathrm{~m}$. The radiation undergoes diffraction in the small gain region; it also experiences rapid focusing due to the gain guiding at the onset of the exponential growth [25, 26], leading to the narrowing of the transverse intensity profile. Therefore, it seems that the transverse profile of the radiation is guided by the exponentially growing amplitude. In order to find the more precise position of the saturation point, rather than using the intensity profile of Fig. 1, we can look at the point where mode narrowing stops and the intensity profile widens. After the saturation point the radiation waist begins to grow as the gain guiding is no longer effective there. 

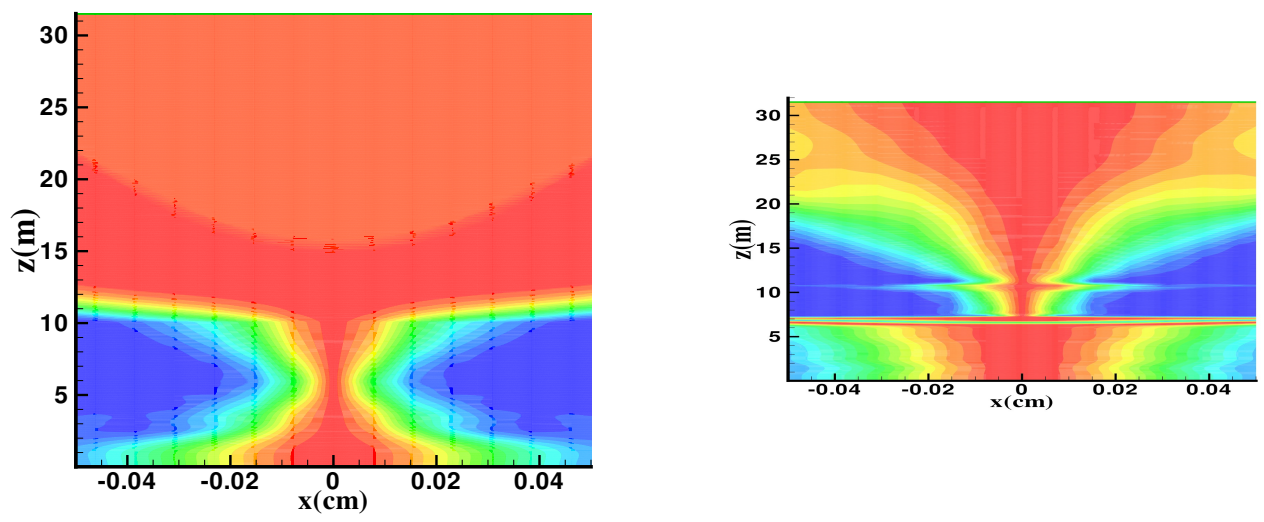

Figure 2: Transverse intensity profile of the fundamental resonance wavelength (a) and the third harmonic wavelength (b) in the $\mathrm{x}$ direction for $y=0$.

Before the saturation point, strong Gaussian profiles with gentle ripples on their outskirts indicate that $T E M_{00}$ is dominant in this region. This result is also shown in Fig. 5 in more detail. However, additional modes tend to grow, beyond the saturation point.

In Fig. 3, the radiation waist of the fundamental resonance and third harmonic are plotted. The radiation waist for the third harmonic is observed to expand, from its initial size, in the small signal region because of vacuum diffraction. This can also be seen in Fig. 2(b). In the exponential growth region, optical guiding becomes strong and focusing is rapid. Finally, the radiation waist expands rapidly as the saturation point is reached. The radiation waist for the fundamental resonance behaves to some extent differently, compared to the third harmonic, and grows faster at the suppression point. Here, the radiation waist of the third harmonic is smaller than that of the fundamental resonance in the exponential growth region but the radiation waist of the third harmonic is larger than that of the fundamental resonance in the exponential growth region in the conventional FEL [29]. The curvature of the phase front, $\alpha$, is shown in Fig. 4. Both fundamental and the third harmonic, which are plane waves at the entrance to the wiggler at $z=0$, deviate from plane waves as radiation travels along the wiggler. The curvature of the phase front of the third harmonic increases abruptly as saturation occurs but for the fundamental resonance, it increases rapidly at the suppression point.

It is interesting to study the composition of the optical modes (effective higher modes ) of the third harmonic. The composition process will introduce higher-order modes. Figure 


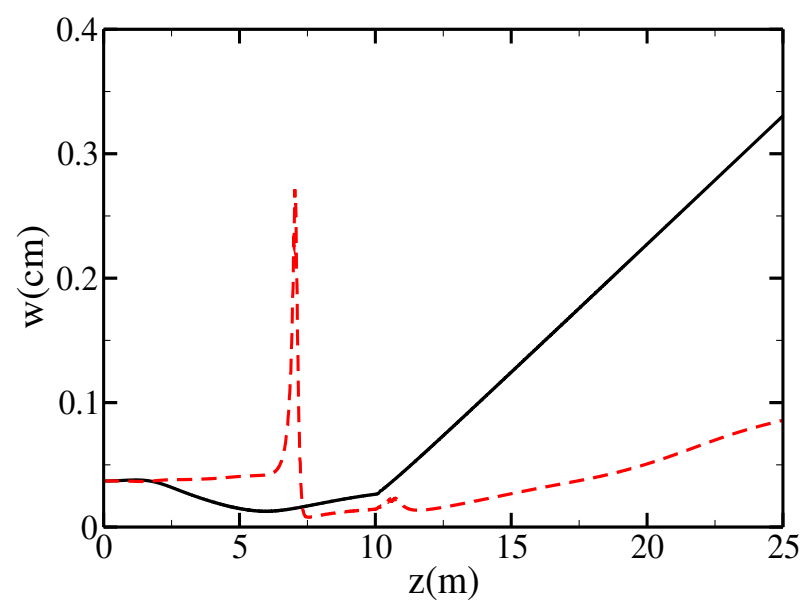

Figure 3: Evolution of the radiation spot size for the fundamental resonance (solid line) and third harmonic (dashed line).

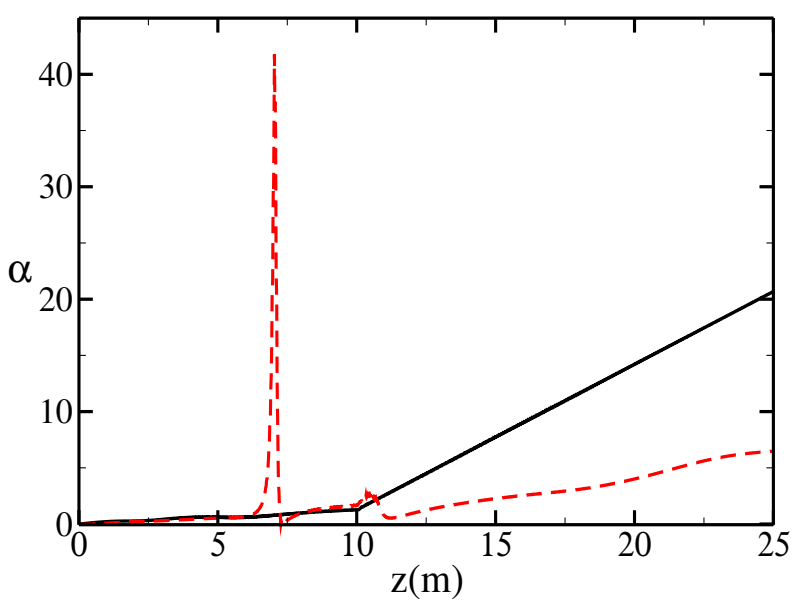

Figure 4: Evolution of $\alpha_{1}$ (solid line) and $\alpha_{3}$ (dashed line) with longitudinal coordinate.

5 shows the mode content histogram at the beginning, suppression and saturation point of the third harmonic. Figure 5 indicates that a purely $(0,0)$ mode exists at the beginning of the wiggler.

Table I] shows the composition of significant modes of the third harmonic at different points of the wiggler. It can be seen that the composition of the lowest order mode $T E M_{0,0}$ is $84.7 \%$ at the suppression point and decreases to $76 \%$ at the saturation point. It is found that the composition is $7 \%$ for either modes $T E M_{20}$ or $T E M_{02}$ at the suppression point and increases to $11 \%$ at the saturation point. Therefore, the lowest order $T E M_{0,0}$ mode is dominant and modes which are shown in Table \have a noticeable contribution and the rest 

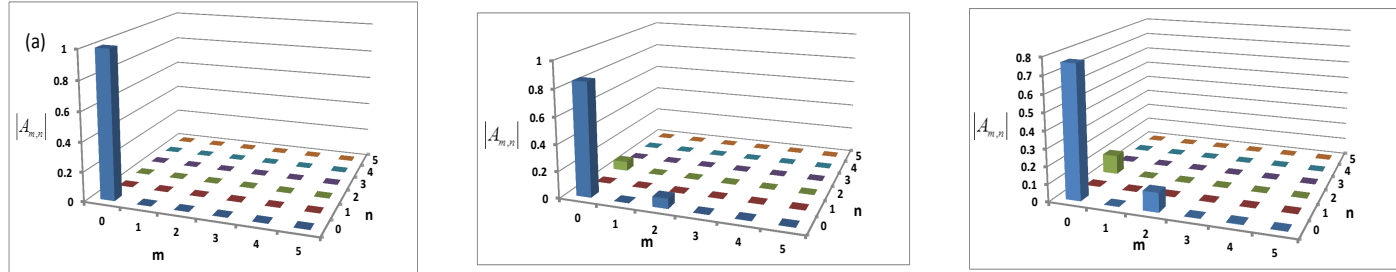

Figure 5: Composition histogram of modes of the third harmonic at the beginning of the wiggler(a), the suppresion point (b), and the saturation point (c).

\begin{tabular}{lccc}
\hline & $\mathrm{z}=0$ & $\mathrm{z}=10 \mathrm{~m}$ & $\mathrm{z}=21.5$ \\
\hline modes & \%composition & \%composition & \% composition \\
\hline$(0,0)$ & $100 \%$ & $84.7 \%$ & $76.3 \%$ \\
$(0,1)$ & $0.0 \%$ & $0.0 \%$ & $0.0 \%$ \\
$(1,0)$ & $0.0 \%$ & $0.001 \%$ & $0.15 \%$ \\
$(0,2)$ & $0.0 \%$ & $7.6 \%$ & $11.1 \%$ \\
$(2,0)$ & $0.0 \%$ & $7.1 \%$ & $11.1 \%$ \\
$(2,2)$ & $0.0 \%$ & $0.11 \%$ & $0.24 \%$ \\
$(0,4)$ & $0.0 \%$ & $0.17 \%$ & $0.36 \%$ \\
$(4,0)$ & $0.0 \%$ & $0.18 \%$ & $0.37 \%$ \\
$(2,4)$ & $0.0 \%$ & $0.004 \%$ & $0.049 \%$ \\
$(4,2)$ & $0.0 \%$ & $0.004 \%$ & $0.049 \%$ \\
$(4,4)$ & $0.0 \%$ & $0.009 \%$ & $0.16 \%$
\end{tabular}

Table I: Modal composition of the third harmonic in different points of the wiggler

are negligible. This higher mode content is clearly illustrated in Fig. 6 where we plot the normalized intensity of the third harmonic near the saturation point.

The thermal effect of the electron beam on optical modes is investigated. Figure 7 shows the evaluation of the normalized amplitude of modes (a) $T E M_{0,0}$, (b) $T E M_{0,2}, T E M_{2,0}$, (c) $T E M_{2,2}$, (d) $T E M_{0,4}, T E M_{4,0}$, and (e) $T E M_{4,4}$ of the third harmonic (solid line) and of the fundamental resonance (dashed line) for initial Gaussian energy spreads of $\sigma_{\gamma} / \gamma=$ 0.0001, 0.001, and 0.004. It is clear from Fig. 7 that by increasing the energy spread, 


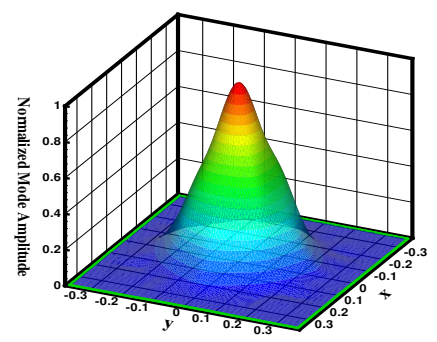

Figure 6: Transverse mode pattern for the third harmonic at the saturation point (for the third harmonic)

(a)

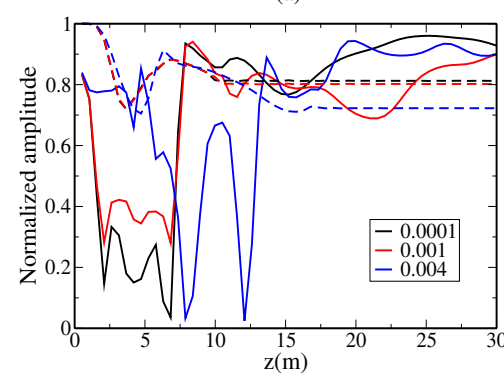

(b)

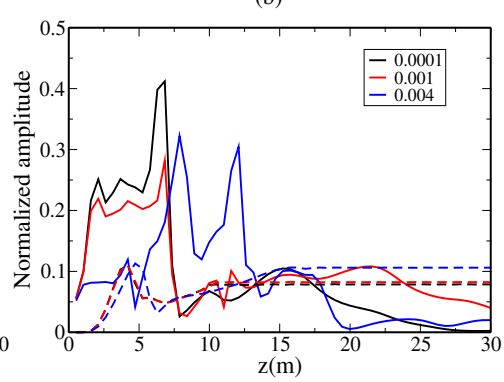

(c)

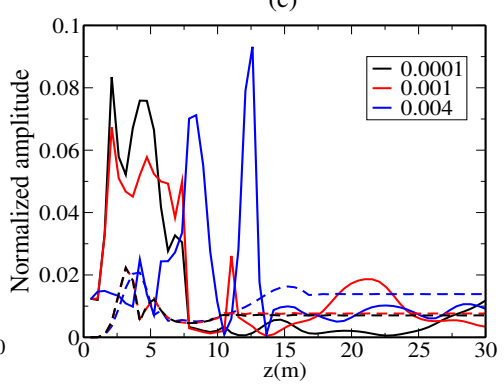

(e)

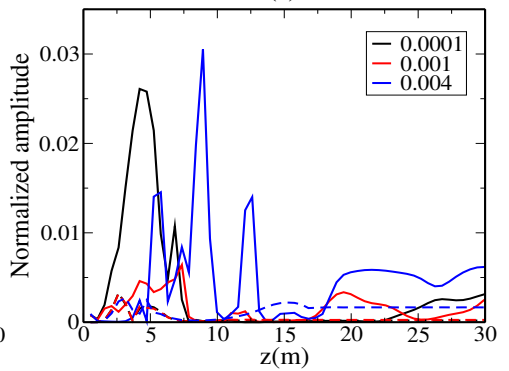

Figure 7: The effect of energy spread on modes of the fundamental resonance and the third harmonic for $\sigma_{\gamma} / \gamma=0.0001,0.001$, and 0.004 .

intensity of the lowest mode decreases but the intensity of higher modes increase at the saturation point. In other words, the share of higher modes increases in higher energy spread compared to that in the low energy spread.

For the shot noise, we apply an appropriate initial condition on the initial phase of macroparticles. Also, we assume a seed power for the fundamental of $0 K W$. The fundamental resonance starts from zero and is suppressed by reducing the wiggler magnetic 


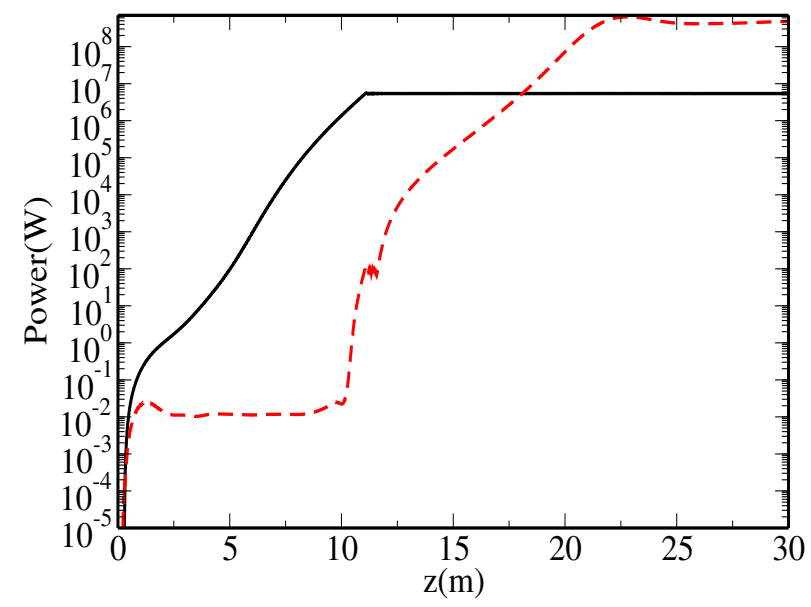

Figure 8: The evolution of the power in the fundamental (solid line) and the third harmonic (dashed line).

field strength at $L_{1}=11 \mathrm{~m}$. In Fig. 8, we plot the evolution of powers in the fundamental and the third harmonic as a function of the distance through the system. It can be seen that powers of the fundamental and the third harmonic initially grow exponentially because of start up of noise. The growth of the fundamental resonance in Fig. 8 is suppressed at $z=11 \mathrm{~m}$ with the power of $5.6 \times 10^{6} \mathrm{~W}$. The small gain regime of the third harmonic starts from $z=1.3 \mathrm{~m}$ to $10 \mathrm{~m}$. After exponential growth regime, it saturates at $z=22.8 \mathrm{~m}$ with the power of $6.3 \times 10^{8} \mathrm{~W}$. We observe that the saturation length and the saturation power of the third harmonic is considerably increased compared to that of the case of seeded FEL. A comparison between the seeded FEL and SASE FEL is shown in Fig. 9 for the conventional FEL. In the conventional FEL, the saturation length of the fundamental resonance in the seeded FEL is lower than that of the SASE FEL.

The transverse intensity profile and the evolution of the radiation waist are presented in Fig. 10 and 11 . The effect of optical guiding due to the electron beam is evident. As it is shown in Fig.10, the transverse intensity is narrower than that of the seeded FEL. Initially, the radiation spot size for the fundamental resonance and the third harmonic is large due to the start up from the shot noise. In the small signal regime, the radiation waist of the third harmonic increases. After that and at the onset of exponential growth, the radiation waist experiences rapid focusing and decreases; it remains small during the exponential growth before expanding at the saturation point . The curvature of the phase front for the third harmonic at the small signal, in Fig. 12 increases abruptly but it decreases at the beginning 


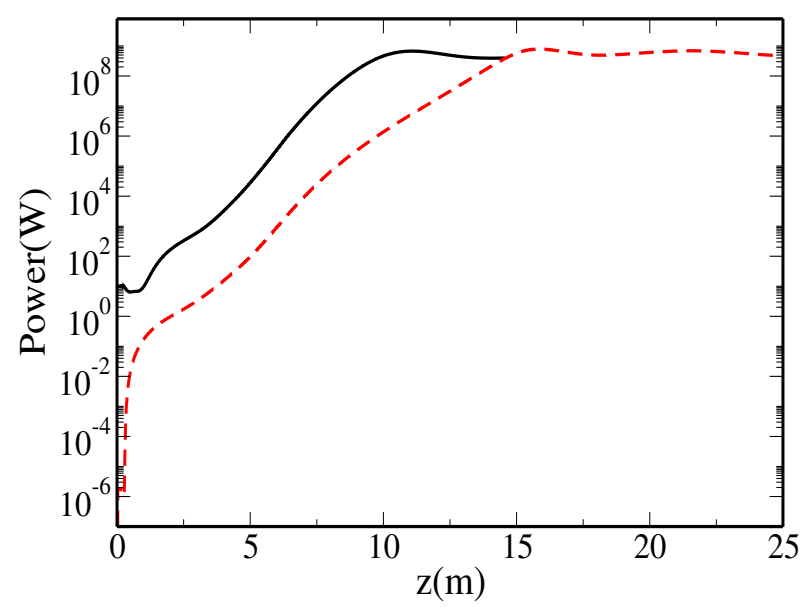

Figure 9: The evolution of the power of the fundamental resonance for seeded FEL (solid line) and SASE FEL (dashed line)
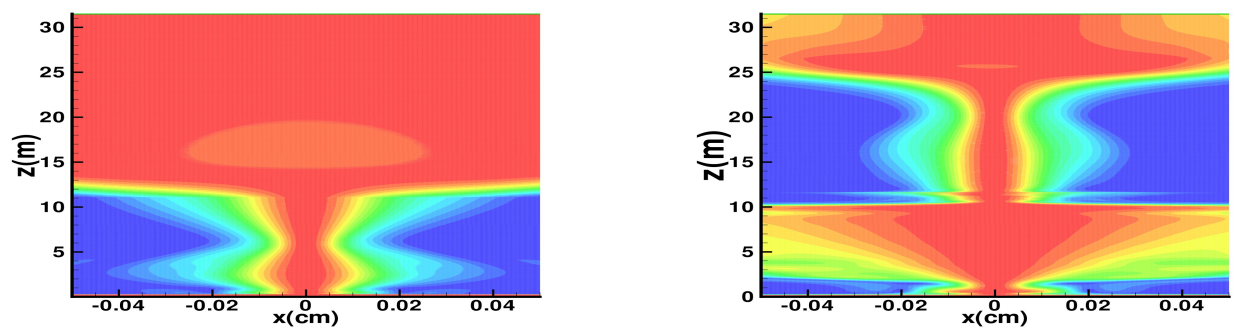

Figure 10: Transverse intensity profile of the fundamental resonance wavelength (a) and the third harmonic wavelength (b) in the y direction for $x=0$

of the exponential growth region and behaves more like a plane wave as it moves on. Also, at the saturation point, the curvature of the phase front is lower $\left(\alpha_{3} \approx 1.5\right)$ than that in the seeded FEL $\left(\alpha_{3} \approx 7\right)$.

The composition of the optical modes of the third harmonic at the beginning, suppression, and saturation point is shown in Fig. 13 and Tabel II It is evident that the composition of the modes at the beginning is low because there is no coherent radiation at the beginning of the wiggler. Also, the composition of the lowest order mode is $84.06 \%$ at the suppression point and increases to $93.86 \%$ at the saturation point. And it is found that compositions for 


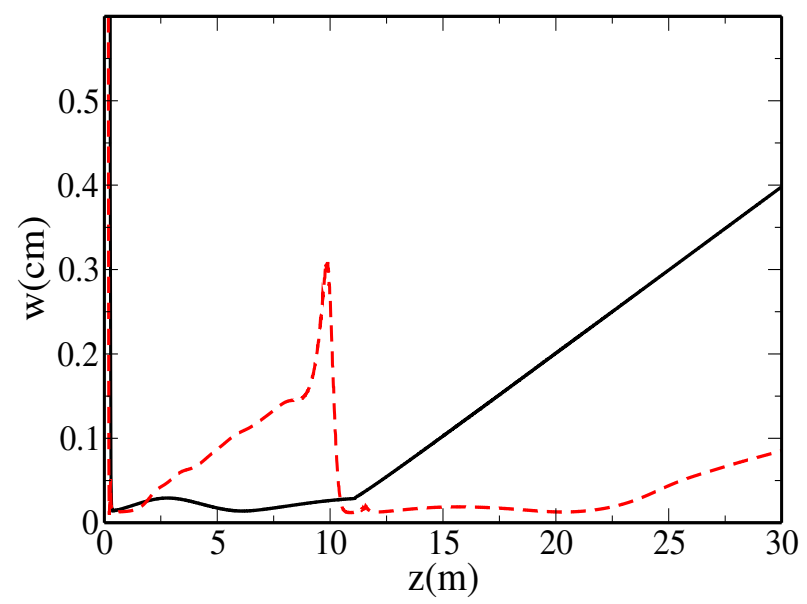

Figure 11: Evolution of the radiation waist for the fundamental resonance (solid line) and third harmonic (dashed line).

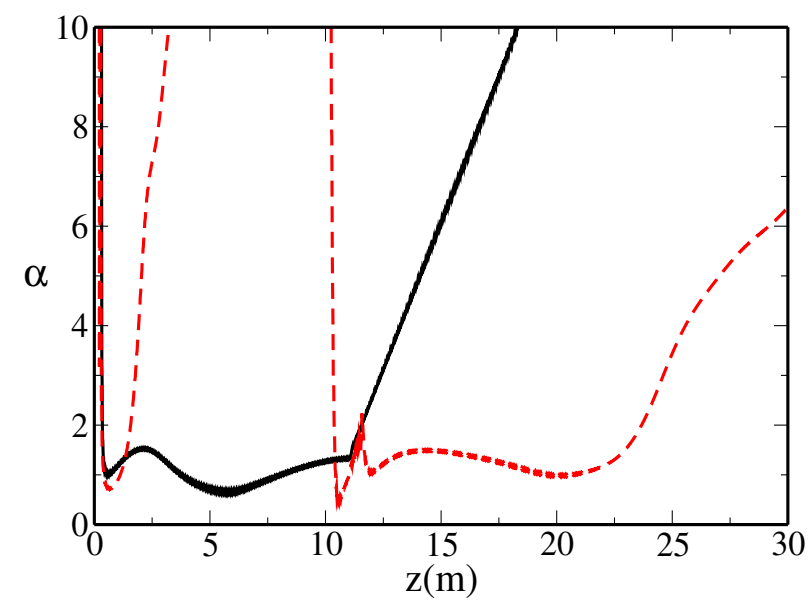

Figure 12: Evolution of $\alpha_{1}$ (solid line)and $\alpha_{3}$ (dashed line) with longitudinal coordinate.

modes $T E M_{20}$ and $T E M_{02}$ are $6.4 \%$ and $5.6 \%$ at the suppression point, respectively, and they decrease to $2.6 \%$ at the saturation point for both modes. It is clear that mode $T E M_{00}$ is dominant at the saturation point, which is reported in Table II] At the saturation point, the composition of the lowest mode in SASE FEL is larger than that of in the seeded FEL and higher modes are less effective in SASE FEL compared to those in the seeded FEL. Because in the SASE FEL, the power of radiation levels off completely at the saturation point. However, the radiation power of seeded FEL has a very small growth at the saturation point, therefor, higher number of modes also contribute to the composition at the saturation point compared to the higher modes of radiation in the SASE FEL. 

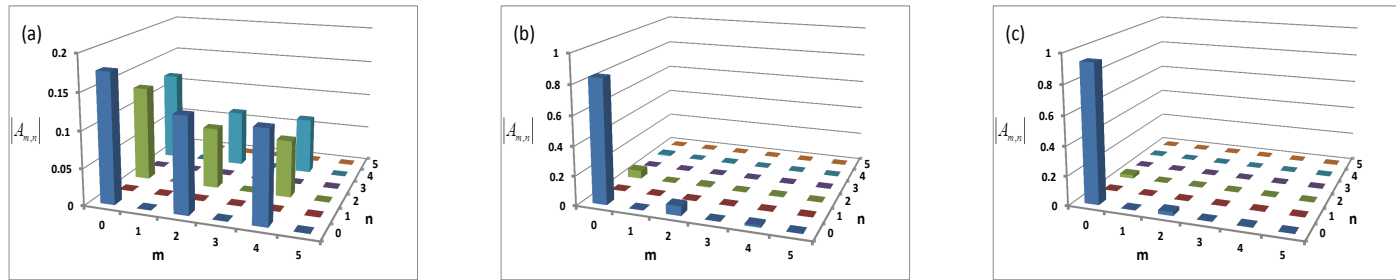

Figure 13: Composition histogram of modes at the beginning of the wiggler(a), the suppresion point (b), and the saturation point (c)

\begin{tabular}{cccc}
\hline & $\mathrm{z}=0$ & $\mathrm{z}=11 \mathrm{~m}$ & $\mathrm{z}=22.8$ \\
\hline modes & \%composition & \% composition & \% composition \\
\hline$(0,0)$ & $17.6 \%$ & $84.06 \%$ & $93.86 \%$ \\
$(0,1)$ & $\approx 0.0 \%$ & $\approx 0.0 \%$ & $\approx 0.0 \%$ \\
$(1,0)$ & $\approx 0.0 \%$ & $\approx 0.0 \%$ & $\approx 0.0 \%$ \\
$(0,2)$ & $12.9 \%$ & $6.4 \%$ & $2.6 \%$ \\
$(2,0)$ & $12.9 \%$ & $5.6 \%$ & $2.6 \%$ \\
$(2,2)$ & $8.2 \%$ & $0.77 \%$ & $0.21 \%$ \\
$(0,4)$ & $12.4 \%$ & $1.3 \%$ & $0.3 \%$ \\
$(4,0)$ & $12.4 \%$ & $1.02 \%$ & $0.3 \%$ \\
$(2,4)$ & $7.8 \%$ & $0.33 \%$ & $0.05 \%$ \\
$(4,2)$ & $7.8 \%$ & $0.29 \%$ & $0.05 \%$ \\
$(4,4)$ & $7.7 \%$ & $0.04 \%$ & $0.016 \%$
\end{tabular}

Table II: Modal composition of the third harmonic in different points of the wiggler

The normalized intensity of the third harmonic near saturation point is represented in Fig. 14.

\section{CONCLUSION}

In this paper, we analyzed detuning of the fundamental to enhance harmonic generation in x-ray FELs. It works well in the frame work of the realistic 3D model of the FEL process by using a nonaveraged simulations, which is named CYRUS 3D. In the absence of slippage 


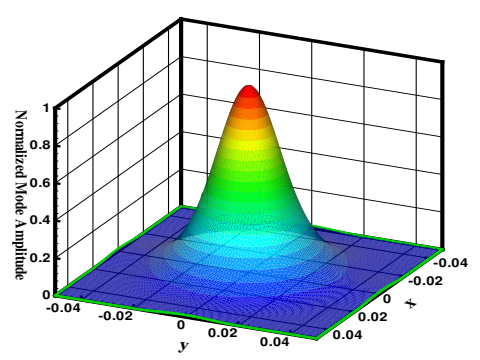

Figure 14: Transverse mode pattern for the third harmonic at the saturation point (for the third harmonic)

(steady-state simulation), the variation of radiation waists, curvatures, and amplitudes for fundamental resonance and the third harmonic are studied. Transverse mode evolution of the fundamental and the third harmonic are investigated in more details. The radiation power of the third harmonic is larger than that of the fundamental resonance in contrast to the nonlinear harmonic generation. Also, the composition of significant modes of the third harmonic is presented which shows that the lowest order mode is dominant. We discussed shot noise in harmonic lasing FEL that treats the start up of both the fundamental and harmonic generation. The saturation power of the third harmonic is increased considerably compared to that of the seeded FEL case while increase of the saturation length is negligible in SASE FEL. Also TEM $M_{00}$ mode is more effective at the saturation point in SASE FEL compared to that in the seeded FEL .

\section{Acknowledgments}

This work was supported by Institute for Research in Fundamental Sciences (IPM) to allow us to use computational facilities.

[1] W. Ackermann, G. Asova, V. Ayvazyan, A. Azima, and N. Baboi, Nature Photon. 1, 336 (2007). 
[2] R. Bonifacio, L. De Salvo, and P. Pierini, Nucl. Instrum.Methods Phys. Res., Sect. A 293, 627 (1990).

[3] H. P. Freund, S. Biedron, and S. Milton, Nucl.Instrum.Methods Phys. Res., Sect. A 445, 53 (2000).

[4] Z. Huang and K. Kim, Phys. Rev. E 62, 7295 (2000).

[5] E. L. Saldin, E. A. Schneidmiller, and M. V. Yurkov, Phys. Rev. ST Accel. Beams 9, 030702 (2006).

[6] W. B. Colson, G. Dattoli, and F. Ciocci, Phys. Rev. A 31, 828 (1985).

[7] G. Dattoli, P. L. Ottaviani, and S. Pagnutti, J. Appl. Phys. 97, 113102 (2005).

[8] L. h. Yu, Phys. Rev. A 44, 5178 (1991).

[9] L. H. Yu, M. Babzien, I. Ben-Zvi, L. F. DiMauron,A. Doyuran,W. Graves,E. Johnson,S. Krinsky, R. Malone,I. Pogorelsky,J. Skaritka, and et al., Science 289, 932 (2000).

[10] A. Doyuran, M. Babzien, T. Shatan, L. H. Yu, L. F. DiMauro,I. Ben-Zvi, S. G. Biedron, W. Graves, E. Johnson, S. Krinsky, R. Malone, and et al., Phys. Rev. Lett. 86, 5902 (2001).

[11] L. Giannessi, M. Bellaveglia, E. Chiadroni, A. Cianchi,ï¡œM. E. Couprie, M. Del Franco, G. Di Pirro, M. Ferrario, G. Gatti, M. Labat, G. Marcus, and et al., Phys. Rev. Lett. 110, 044801 (2013).

[12] M. Labat, M. Bellaveglia, M. Bougeard, B. Carre, F. Ciocci,E. Chiadroni,A. Cianchi,M. E. Couprie,L. Cultrera,M. Del Franco,G. Di Pirro,and et al., Phys. Rev. Lett. 107, 224801 (2011).

[13] G. stupakov, Phys. Rev. Lett.102, 074801 (2009).

[14] G. stupakov, D. Xiang, Phys. Rev. ST Accel. Beams 12, 030702 (2009).

[15] B. W. J. McNeil, G. R. M, Robb, and M. W. Poole, Phys. Rev. E 70, 035501(R)(2004).

[16] M. H. Rouhani and B. Maraghechi, Phys. Rev. ST Accel. Beams 13, 080706(2010).

[17] N. S. Mirian, B. Maraghechi, Phys. Plasmas 21, 043108 (2014).

[18] G. Dattoli, L. Giannessi, P. L. Ottaviani, H. P. Freund, S. G. Biedron, S. Milton, Nucl. Instrum.Methods Phys. Res., Sect. A 495, 48 (2002).

[19] G. Dattoli, M. Galli, P. L. Ottaviani, 1-Dimensional simulation of FEL including high-gain regime, saturation, prebunching and harmonic generation, ENEA Report RT/INN/93/09.

[20] B. W. J. McNeil, G. R. M. Robb, M. W. Poole, and N. R. Thompson, Phys. Rev. Lett. 96, 084801 (2006).

[21] E. A. Schneidmiller and M. V. Yurkov, Phys. Rev. ST Accel. Beams 15, 080702 (2012). 
[22] H. P. Freud, N. A. Yampolsky, and Q. Marksteiner, Phys. Rev. ST Accel. Beams 17, 010702 (2014).

[23] E. Salehi, B. Maraghechi, N. S. Mirian, Phys. Plasmas 22, 033110 (2015).

[24] G. T. Moore, Nucl. Instrum. Methods Phys. Res.Sect. A 239, 19 (1985).

[25] G. Moore, Opt. Commun. 52, 46 (1984).

[26] E. Scharlemann, A. M. Sessler, and J. S. Wurtele, Phys. Rev. lett. 54, 1925 (1986).

[27] S. G. Biedron, H. P. Freund, S. V. Milton, G. Dattoli, A. Renieri, and P. L. Ottaviani, Nucl. Instrum. Methods Phys. Res. Sect. A 528, 443 (2004).

[28] E. L. Saldin, E. A. Schneidmiller, and M. V. Yurkov, Opt. Commun. 281, 1179 (2008).

[29] H. P. Freund, S. G. Biedron, S. V. Milton, IEEE J. Quantum Electron. 36, 275 (2000).

[30] L. H. Yu and J. Wu, Nucl. Instrum. Methods Phys. Res A 483, 493 (2002).

[31] B. Hafizi and C. W. Roberson, Nucl. Instrum.Methods Phys. Res.A 375, 78 (1996).

[32] H. P. Freund, R. C. Davidson, and G. L. Gohnston, IEEE. J. Quantum electron, 27, 2550 (1991).

[33] J. M. Wang and L. H. Yu, Nucl. Instrum. Methods Phys. Res. A 250, 484 (1986).

[34] E. L. Saldin, E. A Schneidmiller, and M. V. Yurkov, Opt. Commun. 148, 383 (1998).

[35] R. Bonifacio, L. De Salvo, P. Pierini, N. Piovella, and C. Pellegrini, Phys. Rev. Lett. 73, 70 (1994).

[36] C. Penman and B. W. J. McNeil, Opt. Commun. 90, 82 (1992).

[37] W. M. Fawley, Phys. Rev. ST Accel. Beams 5, 070701 (2002).

[38] G. Dattoli, L. Mezi, P. L. Ottaviani, and S. Pagnutti, J. Appl. Phys. 99, 044904 (2006).

[39] H. P. Freund, L. Giannessi, and W. H. Miner, Jr. J. Appl. Phys. 104, 123114 (2008).

[40] P. Sprangle, A. Ting, and C. M. Tang, Phys. Rev. Lett. 59, 202 (1987).

[41] B. Hafizi, P. Sprangle, and A. Ting, Phys. Rev. A 36, 1739 (1987).

[42] P. Sprangle, A. Ting, and C. M. Tang, Phys. Rev. A 36, 2773 (1987).

[43] B. Hafizi, P. Sprangle, and J. R. Penano, Nucl. Instrum. Methods Phys. Res.Sect. A 581, 601 (2007).

[44] M. H. Rouhani, E. Salehi, N. S. Mirian, http://particles.ipm.ir/Cyrus1D.jsp

[45] L. Ibanez and S. Johnston, IEEE J. Quantum Electron.19, 339 (1983).

[46] A. Chakhmachi and B. Maraghechi, Phys. Plasmas 16, 043110 (2009).

[47] L. Giannessi, Proceedings of the 26th International Conference on Free-Electron Lasers, 2004, 
P. 37 . 